\title{
Induction of insulin secretion by apolipoprotein M, a carrier for sphingosine 1-phosphate
}

Makoto Kurano, ${ }^{1}$ Masumi Hara, ${ }^{3}$ Koichi Tsuneyama, ${ }^{4}$ Hideyuki Sakoda, ${ }^{2}$ Tomo

Shimizu, ${ }^{5}$ Kazuhisa Tsukamoto, ${ }^{6}$ Hitoshi Ikeda, ${ }^{1,7}$ and Yutaka Yatomi ${ }^{1,7}$

${ }^{1}$ Department of Clinical Laboratory Medicine, ${ }^{2}$ Department of Metabolic Diseases, Graduate School of Medicine, The University of Tokyo, Tokyo, Japan;

${ }^{\mathbf{3}}$ The Fourth Department of Internal Medicine, Teikyo University Mizonokuchi Hospital, Kawasaki, Japan;

${ }^{4}$ Department of Diagnostic Pathology, Graduate School of Medicine and Pharmaceutical Sciences, University of Toyama, Toyama, Japan;

5 Tsukuba Research Institute, Research \& Development Division, Sekisui Medical Co., Ltd., Ibaraki, Japan;

${ }^{6}$ Department of Metabolism, Diabetes and Nephrology, Aizu Medical Center, Fukushima Medical University, Fukushima, Japan;

${ }^{7}$ Department of Clinical Laboratory, The University of Tokyo Hospital, Tokyo, Japan.

Email addresses:

MK: kurano-tky@umin.ac.jp; MH: masumihara-tky@umin.ac.jp;

KT: ktsune@med.u-toyama.ac.jp; HS: hsakoda-tky@umin.ac.jp;

TS: shimizu154@ sekisui.com; KT: kazut@fmu.ac.jp;

HI: ikeda-1im@h.u-tokyo.ac.jp; YY: yatoyuta-tky@umin.ac.jp

Address correspondence to:

Yutaka Yatomi, $\mathrm{MD}, \mathrm{PhD}$,

Department of Clinical Laboratory Medicine, Graduate School of Medicine,

The University of Tokyo, 7-3-1 Hongo, Bunkyo-ku, Tokyo 113-8655, Japan.

Tel: +81-3-5800-8721;

Fax: +81-3-5689-0495;

e-mail: yatoyuta-tky@umin.ac.jp 


\begin{abstract}
Backgrounds: High-density lipoprotein (HDL) has been proposed to enhance $\beta$-cell functions. Clinical studies have suggested that apolipoprotein $\mathrm{M}$ (apoM), which rides mainly on HDL, is involved in diabetes; however, the underlying mechanism has not yet been elucidated. Recently, apoM was shown to be a carrier for sphingosine 1-phosphate (S1P), a bioactive lipid mediator. In the present study, we investigated the modulation of insulin secretion by apoM through the action of S1P.
\end{abstract}

Methods and Results: We overexpressed apoM in the livers of C57BL6 mice using adenovirus gene transfer and found that the blood glucose levels under ad libitum feeding conditions were lower in the apoM-overexpressing mice. While an insulin tolerance test revealed that insulin sensitivity was not significantly affected, a glucose tolerance test revealed that apoM-overexpressing mice had a better glucose tolerance because of enhanced insulin secretion, a phenomenon that was reversed by treatment with VPC 23019, an antagonist against S1P1 and S1P3 receptor. In vitro experiments with MIN6 cells also revealed that apoM-containing lipoproteins enhanced insulin secretion, which was again inhibited by VPC 23019. ApoM retarded the degradation of S1P, and an increase in Pdx1 expression, the attenuation of endoreticulum stress, and the phosphorylation of Akt, AmpK, and Erk were observed as possible underlying mechanisms for the effect of S1P, maintained at a high concentration by apoM, on the increase in insulin secretion.

Conclusions: ApoM augmented insulin secretion by maintaining the S1P concentration under both in vivo and in vitro conditions.

Keywords: apolipoprotein M, sphingosine 1-phosphate, insulin secretion, MIN6 cells 


\section{Abbreviations}

ApoA-I apolipoprotein A-I

ApoM apolipoprotein M

ApoM-Lps apoM-containing lipoproteins prepared from CM-apoM

CM-null conditional media of HepG2 cells infected with blank adenovirus

CM-apoM conditional media of HepG2 cells infected with adenovirus coding apoM

ER endoreticulum

HDL high-density lipoprotein

Null-Lps control lipoproteins prepared from CM-null

S1P sphingosine 1-phosphate

VPC $\quad$ VPC 23019 


\section{Introduction}

Diabetes mellitus threatens the lives of people all around the world. The underlying mechanism for type 1 diabetes is $\beta$-cell death, while that for type 2 diabetes is impaired insulin secretion from $\beta$-cells accompanied by increased insulin resistance as a result of obesity. Therefore, elucidating the factors that influence insulin secretion from $\beta$-cells is an important issue for the treatment and diagnosis of diabetes.

So far, many factors, such as insulin, incretins, fatty acids, cytokines, and so on, have been shown to influence the functions of $\beta$-cells [1]. Among them, high-density lipoprotein (HDL) has recently been discovered to possess protective properties for $\beta$-cell functions: HDL prevents $\beta$-cell death, promotes $\beta$-cell proliferation, and enhances insulin secretion from $\beta$-cells [2]. The mechanism responsible for these beneficial effects of HDL can mainly be attributed to the ability of HDL to promote cholesterol efflux: HDL improves $\beta$-cell dysfunction by attenuating cholesterol loading [3]. Along with its ability to cause the efflux of cholesterol, HDL possesses several pleiotropic effects, such as antioxidant $[4,5]$ and anti-apoptotic properties $[6$, 7]. These protective effects of HDL are thought to arise from several minor proteins that bind to HDL [8] other than apoA-I, the main component protein of HDL; however, the HDL substrates that promote $\beta$-cell functions have not yet been elucidated.

Among these proteins that bind to HDL, apolipoprotein $\mathrm{M}$ (apoM), a minor apolipoprotein [9], has been reported to be involved in diabetes; SNPs of apoM have been 
shown to be related to type 1 diabetes [10] and type 2 diabetes [11, 12]; however, the underlying mechanisms for these epidemiological relations have not yet been elucidated. Regarding the physiological properties of apoM, apoM has been shown to be involved in pre- $\beta$ HDL formation and to have atheroprotective effects in animal experiments [13] [14]. Along with this role in HDL formation, apoM was recently reported to be a carrier of sphingosine 1-phosphate (S1P), a bioactive lipid mediator [15]. S1P has been shown to exert important roles in various fields, such as vascular biology, oncology, immunology and embryology, and is responsible, at least in part, for the pleiotropic effects of HDL $[16,17]$. This finding prompted us to investigate the hypothesis that apoM might modulate glucose metabolism through S1P signaling.

In the present study, to investigate how apoM modulates glucose metabolism and to determine whether S1P may be involved in these modulations, we overexpressed apoM in wild-type mice with or without treatment with VPC 23019 (VPC), an antagonist against S1P1 and S1P3. We also investigated the effects of the conditional media of apoM-overexpressing HepG2 cells on MIN6 cells, a murine insulinoma cell line. 


\section{Materials and methods}

\subsection{Reagents}

Sphingosine 1-phosphate, D-erythro (S1P; Enzo Life Sciences, Plymouth Meeting, PA) was dissolved in methanol. Immediately before use, the methanol was evaporated and the reagents were resolved in apoM-Lps, null-Lps, recombinant apoM (R\&D Systems, Minneapolis, MN), or PBS containing $0.4 \%$ fatty acid-free BSA (Sigma-Aldrich Co., St. Louis, MO). VPC 23019 (VPC; Avanti Polar Lipids, Alabaster, AL) was dissolved in DMSO for the in vitro experiments; for the in vivo experiments, VPC was dissolved in ethanol and the ethanol was evaporated just before use, then dissolved again in PBS containing $0.4 \%$ fatty acid-free BSA.

\subsection{Animal experiments}

Ten-week-old C57BL6J mice were obtained from CLEA Japan (Tokyo, Japan) and were infected with adenovirus coding apoM or control blank adenovirus at a dose of $2.5 \times 10^{8}$ pfu/g body weight via the mouse tail vein, as described in a previous report [18]; the analyses were performed five days later.

For the insulin tolerance test, mice were subjected to a fast for 6 hours and then challenged with Humulin R (0.5 U/kg body weight; Ely Lilly, Indianapolis, IN) intraperitoneally. The blood glucose level was measured before the administration of insulin and at 20, 40, 60, 90, 
and 120 minutes after administration. For the glucose tolerance test, mice were challenged with the vehicle alone or with VPC $(0.75 \mathrm{mg} / \mathrm{kg}$ body weight $)$ once a day from the second day after the viral administration; the mice were then subjected to a fast for 6 hours and challenged with glucose (2 g/kg body weight) intraperitoneally (Supplemental Figure 1A). Blood glucose was measured before and at 20,40,60, 90, and 120 minutes after the administration of glucose, and plasma samples for the insulin measurement were collected before and at 20,40, and 60 minutes after administration.

\subsection{Analysis of blood glucose and plasma insulin levels}

The blood glucose levels were measured using a Glutest sensor (Sanwa Kagaku, Nagoya, Japan). The plasma insulin levels were determined using an insulin enzyme-linked immunosorbent assay (ELISA) kit (Sibayagi, Gunma, Japan).

\subsection{Cell experiments}

MIN 6 cells were originally received from Dr. Miyazaki [19] and were cultured in

DMEM (Sigma-Aldrich Co.) supplemented with 10\% fetal bovine serum (FBS; Gibco BRL,

Eggstein, Germany) and 1\% penicillin/streptomycin (Gibco BRL). 
ApoM-containing lipoproteins (apoM-Lps) and control lipoproteins (null-Lps) were prepared from the conditional media of apoM-overexpressing HepG2 cells (CM-apoM) or control conditional media (CM-null) (prepared as described previously[18]); the total volumes of CM-apoM or CM-null were $12 \mathrm{~mL} / \mathrm{dish}$ and were concentrated to about $500 \mu \mathrm{L} / \mathrm{dish}$ by centrifugation and purification using Amicon Ultra-15 (UFC901008, Millipore Co., Bedford, MA). ApoM-Lps and null-Lps were added to FCS-free DMEM at the ratio of 1:9.

To examine the effects of apoM-Lps on insulin secretion from MIN6 cells, after incubation in the media for 4 hours or 16 hours, the media were pre-incubated in glucose-free HEPES-Krebs buffer (119 mM NaCl, $4.74 \mathrm{mM} \mathrm{KCl,} 2.54 \mathrm{mM} \mathrm{CaCl} 2,1.19 \mathrm{mM} \mathrm{MgCl} 2,1.19$ $\mathrm{mM} \mathrm{KH}_{2} \mathrm{PO}_{4}, 25 \mathrm{mM} \mathrm{NaHCO}_{3}$ and $10 \mathrm{mM}$ HEPES [pH7.4]) containing apoM-Lps or null-Lps with or without S1P for 30 minutes. At this time, the concentration of S1P corresponded to the concentration of S1P after the incubation of MIN6 cells for 4 hours or 16 hours (Figure 3B). Subsequently, the medium was replaced with HEPES-Krebs buffer containing $20 \mathrm{mM}$ glucose, and the cells were further incubated for 10 minutes. The medium was collected and diluted 1:50 to measure the insulin level using an insulin enzyme-linked immunosorbent assay (ELISA) kit (Sibayagi). The insulin concentrations were then adjusted according to the amount of cell protein. A schema showing the protocol for the cell experiments is shown in Supplemental Figure 1B. 
To investigate the effects of S1P bound to recombinant apoM, similar experiments were performed with $500 \mathrm{nM}$ recombinant apoM or fatty acid free albumin as a vehicle.

\subsection{Human apoM ELISA}

Human apoM ELISA was developed as follows; 96-well plates (Thermo Fisher Scientific Inc. Allentown, PA) were coated with $250 \mathrm{ng} /$ well of goat anti-mouse IgG (Jackson ImmunoResearch Laboratories Inc. West Grove, PA) in PBS, followed by blocking with PBST containing 1\% BSA. Then, mouse anti-human apoM monoclonal antibody developed against recombinant human apoM (ATGen Co., Ltd. Gyeonggi-do, Korea) added to the plate, followed by incubation for 1 hour. The samples were denatured with PBS containing 2\% SDS and 0.2M DTT (pH7.2), diluted in PBST containing skimmed milk (1:600) and added on the plate. After 1 hour, the samples were replaced for biotinylated rabbit anti-human apoM antibody (developed against the C-terminal peptide of human apoM). Human apoM bound to the plate was detected with horseradish peroxidase-labeled streptavidin and $o$-phenylenediamine (OPD). A dilution series of standard made from a serum pool from healthy subjects was included on each plate to calculate the apoM concentration.

The variation coefficient of the assay was $2.8 \%$ and the standard curve for ELISA measurements of diluted human serum pool was shown in Supplementary Figure 2.

\subsection{Measurement of S1P}

The S1P contents in the plasma and in the conditional media were determined using a two-step lipid extraction process followed by HPLC separation, as described previously [20]. 


\subsection{Real-time PCR}

The total RNA was extracted from the MIN6 cells using a GenElute mammalian total RNA miniprep kit (Sigma-Aldrich) and then subjected to reverse transcription with Superscript II enzyme (Invitrogen Co. Carlsbad, CA). Quantitative PCR was performed using an ABI 7300 Real-Time PCR System (Applied Biosystems) for Pdx1 (Mm 00435565_m1), Grp78 (Mm00517690_g1) and GAPDH (Mm 99999915_g1). The expression levels of Pdx1 and Grp78 were adjusted to those of the endogenous GAPDH mRNA control.

\subsection{XBP-1 splicing assay}

An XBP-1 splicing assay was performed as described previously [21]. Briefly,

following the PCR-amplification of XBP-1 cDNA, which included the region where the ER stress induced the processing of mRNA, the PCR products were digested with PstI and were analyzed with electrophoresis using a 2\% agarose gel. The processing of XBP-1 mRNA with ER stress resulted in the elimination of a PstI cutting site, forming high molecular bands on the electrophoretic gel. 


\subsection{Western blotting}

Murine livers, skeletal muscles (soleus muscles), and cell samples were prepared using RIPA lysis buffer (25 mM Tris HCL [pH7.6], 1\% NP-40, 0.1\% SDS, $150 \mathrm{mM} \mathrm{NaCl}, 0.02 \%$ sodium deoxycholate, $1 \mathrm{mM}$ orthovanadate, $1 \mathrm{mM}$ PMSF, and protease inhibitor cocktail [Roche, Mannheim, Germany]). Western blotting was performed as a protein analysis using the following antibodies: anti-Erk and anti-phospho Erk antibody (BD Bioscience, San Jose, CA); anti-Akt, anti-phospho Akt, anti-AMPK, and anti-phospho-AMPK antibody (Cell Signaling Technology, Danvers, MA); and anti-apoM serum, developed as described previously [18].

\subsection{Statistical analysis}

The results were expressed as the mean \pm SEM. Differences between the two groups were evaluated using a Student $t$-test, and differences among more than two groups were assessed using a one-way ANOVA, followed by multiple comparison tests. P-values less than 0.05 were deemed statistically significant. 


\section{Results}

\subsection{Hepatic apoM overexpression in wild-type mice prevented the increase in blood glucose}

\section{levels after ad libitum feeding}

We overexpressed apoM in wild-type mice using adenovirus gene transfer and examine the effects of apoM overexpression on glucose metabolism in vivo (Figure 1A). As described previously [18], the plasma S1P levels were increased by apoM overexpression (Figure 1B). Regarding the blood glucose levels, although the blood glucose level after 6 hours of fasting was not significantly modulated by apoM overexpression, the ad libitum blood glucose levels were significantly lower in apoM-overexpressing mice (Figure 1C). To investigate the underlying mechanisms, we first performed an insulin tolerance test to evaluate the modulation of insulin sensitivity; however, no significant difference was observed between the apoM-overexpressing mice and the control mice (Figure 1D), while the Akt phosphorylation in the liver and skeletal muscle was not augmented by apoM overexpression under a fasting condition (Figure 1E and F). The expression of any genes involved in hepatic glucose metabolism was not significantly decreased in apoM-overexpressing mice, either (Supplementary Figure 3A). Although the expression of G6Pase and PGC1a, which are involved in gluconeogenesis, tended to be decreased, the result of pyruvate tolerance test suggests that the suppression of gluconeogenesis might not mainly explain the glucose-lowering 
effect by apoM (Supplementary Figure 3B and C). These results suggest that the modulation of insulin sensitivity might not be involved in the improvement of glucose metabolism through apoM overexpression, at least in wild-type mice.

\subsection{Hepatic apoM overexpression in wild-type mice enhanced insulin secretion and}

\section{ameliorated glucose tolerance}

Next, we investigated the possibility that apoM might modulate insulin secretion through S1P, because S1P has been reported to enhance $\beta$-cell functions in vitro $[22,23]$ and apoM has been elucidated to be a carrier of S1P [15]. We treated apoM-overexpressing mice with VPC 23019 (VPC, an antagonist against S1P1 and SIP3 receptor) to investigate whether S1P signaling was involved in the modulation of glucose metabolism by apoM. As shown in Figure 2A, VPC partially reversed the prevention of the increase in blood glucose levels after ad libitum feeding that was observed in apoM-overexpressing mice. These data suggest that apoM prevents the increase in the blood glucose level after ad libitum feeding thorough S1P.

We next performed a glucose tolerance test to evaluate the modulation of insulin secretion. As shown in Figure 2B and C, apoM overexpression augmented glucose tolerance, while VPC partially reversed this effect. Regarding the insulin levels, although the plasma insulin levels before the administration of glucose were not altered, the plasma insulin levels at 
20 min after the administration of glucose and the area under curve (AUC) of the plasma insulin levels were significantly increased in apoM-overexpressing mice, and this augmentation was inhibited by treatment with VPC (Figure 2D and E). Therefore, the decrease in blood glucose in the ad libitum-fed apoM-overexpressing mice might be due to the increase in insulin secretion by an S1P-mediated pathway.

\section{3. apoM-Lps enhanced insulin secretion from MIN 6 cells}

We investigated whether enhanced insulin secretion, as observed in apoM-overexpressing mice, might also be seen in vitro. We prepared the media using apoM-containing lipoproteins (apoM-Lps) or control lipoproteins (null-Lps) prepared by filtering the conditional medium of HepG2 cells infected with Ad-apoM or Ad-Null, as described in the Materials and Methods section. As shown in Figure 3A, the apoM level was much higher in apoM-Lps, while no obvious difference was observed for albumin and apoA-I.

The time course for the apoM and the S1P levels in these media containing apoM-Lps or null-Lps with or without exogenously added S1P used to culture MIN 6 cells is shown in Figure 3B and 3C, demonstrating that the S1P levels in the media containing apoM-Lps were maintained, compared with those in the media containing null-Lps. 
First, we investigated the modulation of insulin secretion from MIN6 cells by

apoM-Lps. As shown in Figure 3D, at 4 hours after the replacement of the media, S1P at a concentration of $500 \mathrm{nM}$ significantly increased insulin secretion, while apoM-Lps increased insulin secretion to a much larger extent. Moreover, VPC inhibited apoM-Lps- or $500 \mathrm{nM}$ S1P-induced insulin secretion from MIN6 cells, as observed in vivo. At 16 hours, apoM-Lps increased insulin secretion from MIN6 cells, though 500 nM S1P in the medium containing null-Lps did not alter the insulin secretion (Figure 3E). Therefore, in vitro, apoM-Lps might also augment insulin secretion from MIN6 cells, depending on S1P signaling.

\subsection{ApoM-Lps augmented the phosphorylation of Akt, AmpK and Erk in MIN 6 cells}

We next investigated the underlying mechanism for enhanced insulin secretion. The insulin signal itself is known to be important for glucose-stimulated insulin secretion from $\beta$-cells [24] [25], and S1P signal pathways have been demonstrated to share some signaling with the insulin signal, such as the phosphorylation of Akt, AMPK, and Erk [26]. As shown in Figure 4A and B, apoM-Lps significantly phosphorylated Akt to a greater extent than null-Lps and maintained the phosphorylation of Akt for a longer time than when incubated with $500 \mathrm{nM} \mathrm{S1P}$ in medium containing null-Lps. The phosphorylation of Akt was inhibited by VPC at both 4 hours and 16 hours (Figure 4C-F). 
Regarding the phosphorylation of AmpK and Erk, apoM-Lps increased the

phosphorylation of both AmpK and Erk at 4 hours to an extent similar to that of $500 \mathrm{nM} \mathrm{S1P}$

(Figure 5A, B, Supplemental Figure 4A, B), while at 16 hours, only apoM-Lps increased the phosphorylation of AmpK (Figure 5C, D, Supplemental Figure 4C, D).

These results suggest that apoM might enhance $\beta$-cell functions, at least in part,

through the increment of some signal pathway shared with insulin signaling.

\subsection{ApoM-Lps increased Pdx-1 expression in MIN6 cells}

We next investigated the effect of apoM-Lps on Pdx-1 expression, an important

protein in $\beta$ cell function and proliferation [27]. As shown in Figure 6, apoM-Lps increased Pdx protein levels and mRNA levels significantly at both 4 hours and 16 hours, and VPC inhibited these effects, although $500 \mathrm{nMS1P}$ increased the Pdx mRNA levels only at 4 hours.

\subsection{ApoM-Lps attenuated ER stress in MIN6 cells-}

Recently, endoreticulum (ER) stress has been proposed to be involved in determining $\beta$-cell functions [28, 29], and HDL has been reported to attenuate ER stress in $\beta$-cells [7]. Therefore, we measured ER stress by investigating the splicing of XBP-1 and the expression of Grp78, a chaperone induced by ER stress, and found that $500 \mathrm{nM} \mathrm{S1P}$ and apoM-Lps decreased 
spliced XBP-1 and increased non-spliced XBP-1 (Figure 7A and B) and decreased Grp78

mRNA levels (Figure 7C) at 4 hours, suggesting that apoM-Lps attenuates ER stress in MIN6

cells, although these effects on ER stress at 16 hours did not reach statistical significance

(Supplemental Figure 5).

\subsection{Recombinant apoM retarded the degradation of S1P and augmented S1P-mediated}

\section{insulin secretion from MIN6 cells}

Finally, we investigated whether recombinant apoM augmented S1P-mediated insulin

secretion from MIN6 cells. As shown in Figure 8A, when S1P was bound to recombinant apoM,

the S1P levels in the media were maintained to a greater extent than those when S1P was bound

to albumin, although recombinant apoM itself did not affect the S1P contents in the media.

Regarding insulin secretion from MIN6 cells, recombinant apoM itself did not increase insulin secretion, while S1P bound to recombinant apoM augmented insulin secretion more than when S1P was bound to albumin (Figure 8B), suggesting that S1P bound to apoM, but not apoM itself, might increase insulin secretion. 


\section{Discussion}

HDL has been demonstrated to exert various beneficial effects, other than its role in the cholesterol reverse transport system [30] [31]. For example, HDL protects $\beta$-cells from impaired insulin secretion [2]. Among these effects, apoM is a possible candidate as a modulator of HDL functions. Reportedly, ApoM is an important biomarker in diabetes based on clinical studies [10-12]; however, the underlying mechanism has not yet been elucidated.

Recently, apoM has been shown to be a carrier of S1P [15]. This finding prompted us to challenge the hypothesis that apoM might modulate $\beta$-cell functions through S1P signaling. Actually, S1P has been proved to be involved in various fields of diseases $[16,17,32]$; regarding diabetes, $\mathrm{S} 1 \mathrm{P}$ has been reported to enhance $\beta$-cell functions in vitro [23, 33]. However, the role of S1P has been difficult to demonstrate in vivo because S1P is continuously produced and is rapidly degraded in vivo. In this study, to investigate our hypothesis, we overexpressed apoM in mouse livers. Consistent with the results of a previous article [18], the plasma S1P levels increased dramatically (Figure 1B), and we found that the blood glucose level after ad libitum feeding was lower in apoM-overexpressing mice (Figure 1C). The main underlying mechanism was not the augmentation of insulin sensitivity (Figure 1D-F), but an increment in insulin secretion (Figure 2). VPC, an antagonist against S1P1 and S1P3 receptor, inhibited the enhancement of insulin secretion by apoM, suggesting that apoM might increase insulin 
secretion depending on S1P signaling. To our knowledge, this is the first study to elucidate the role of apoM and S1P in the modulation of insulin secretion in vivo.

Similar results were obtained in an in vitro experiment utilizing the apoM-containing lipoproteins (apoM-Lps) prepared by filtering the conditional media of apoM-overexpressing HepG2 cells (Figure 3C and D). The underlying mechanisms through which S1P increases insulin secretion from MIN6 cells have not been completely elucidated, but several biological modulations in MIN6 cells related with insulin secretion were observed by treatment with apoM-Lps in this study. In the first place, Akt, AMPK, and Erk, which are phosphorylated upon insulin stimulation and are thought to be involved in $\beta$-cell functions [25], were phosphorylated by apoM-Lps (Figure 4, Figure 5, and Supplemental Figure 4). These results regarding the modulation of the phosphorylation of Akt, AMPK, and Erk seem to be reasonable based on results obtained in cell lines other than MIN6 cells, in which S1P was shown to activate these proteins and to promote cell proliferation thorough S1P1, which was coupled with Gi protein [26]. To investigate whether we could observe the phosphorylation of these kinases in vivo, we subjected pancreas islets from apoM-overexpressing or control mice to immunohistological staining and found that apoM overexpression somehow increased the phosphorylation of Akt, AmpK, while Erk and VPC treatment restored the phosphorylation (Supplemental Figure 6); however, the phosphorylation seems to spread throughout the islets, and not just the $\beta$-cells, 
suggesting that the effects of apoM and S1P might not be confined to $\beta$-cells, but might also be observed in other components of the pancreas islets. Furthermore, the expression of Pdx-1, a transcription factor necessary for $\beta$-cell maturation [27], was increased by apoM-Lps and was inhibited by treatment with VPC, while ER stress, which increases as a result of demands for more insulin because of insulin resistance and leads to $\beta$-cell loss [28, 29], was attenuated by apoM-Lps. Based on these beneficial effects of apoM-Lps on $\beta$-cell function and proliferation, apoM might protect against diabetes from the aspect of insulin secretion. Interestingly, apoM, in turn, has been demonstrated to be regulated by insulin [34, 35]. This cross-talk between apoM and insulin suggests that apoM might play an important role in maintaining glucose homeostasis; when $\beta$-cell functions were disturbed and insulin levels were lowered, apoM levels in the liver increased; this compensatory increment of apoM resulted in an increase in the S1P level and protected $\beta$-cell functions.

Another interesting finding was that S1P bound to apoM-Lps or recombinant apoM might exert greater biological effects on insulin secretion than S1P bound to albumin. These differences might result from the different time courses of the S1P concentrations; apoM retarded S1P degradation (Figure 3B and 8A). The difference between HDL-linked S1P and albumin-linked S1P has been reported in several previous studies [36, 37] in the field of vascular biology, and the results from the present study suggest that these differences might 
come from the fact that the S1P concentration is well maintained when S1P is bound to apoM.

Therefore, although albumin has been so far utilized as a vehicle in most in vitro studies on S1P biology, the results of the present study suggest that the usage of apoM as a vehicle might be preferable for investigations of the physiological roles of S1P.

Insulin resistance is another important factor in the development of diabetes, along with impaired insulin secretion. Regarding insulin resistance, as shown in Figure 1D-F, apoM did not significantly ameliorate insulin sensitivity in wild-type mice. However, we could not rule out the possibility that apoM improves insulin resistance through S1P signaling in insulin-resistant or diabetic mouse models. Actually, in an in vitro experiment utilizing a skeletal muscle cell line, S1P was shown to ameliorate insulin resistance [38]. Further studies using diabetic mouse models are necessary to investigate this possibility.

In summary, we have demonstrated that apoM increases insulin secretion, depending on S1P signaling. HDL (apoM)-linked S1P might promote insulin secretion both in vivo and in vitro. 


\section{Acknowledgement}

This research was supported by a Grant-In-Aid for Scientific Research (A) No.

22249017 and 25253040 (Y.Y.) and a Grant-in-Aid for Young Scientists (B) No. 25860740

(M.K.) from the Japan Society for the Promotion of Science and by CREST from JST. 


\section{References}

[1] K.J. Chang-Chen, R. Mullur, E. Bernal-Mizrachi, Beta-cell failure as a complication of diabetes, Rev Endocr Metab Disord, 9 (2008) 329-343.

[2] C. Mineo, P.W. Shaul, Novel biological functions of high-density lipoprotein cholesterol, Circ Res, 111 (2012) 1079-1090.

[3] M.A. Fryirs, P.J. Barter, M. Appavoo, B.E. Tuch, F. Tabet, A.K. Heather, K.A. Rye, Effects of high-density lipoproteins on pancreatic beta-cell insulin secretion, Arterioscler Thromb Vasc Biol, 30 (2010) 1642-1648.

[4] M. Koren-Gluzer, M. Aviram, E. Meilin, T. Hayek, The antioxidant HDL-associated paraoxonase-1 (PON1) attenuates diabetes development and stimulates beta-cell insulin release, Atherosclerosis, 219 (2011) 510-518.

[5] R.P. Dullaart, W. Annema, J.F. de Boer, U.J. Tietge, Pancreatic beta-cell function relates positively to HDL functionality in well-controlled type 2 diabetes mellitus, Atherosclerosis, 222 (2012) 567-573.

[6] S. Rutti, J.A. Ehses, R.A. Sibler, R. Prazak, L. Rohrer, S. Georgopoulos, D.T. Meier, N.

Niclauss, T. Berney, M.Y. Donath, A. von Eckardstein, Low- and high-density lipoproteins modulate function, apoptosis, and proliferation of primary human and murine pancreatic beta-cells, Endocrinology, 150 (2009) 4521-4530. 
[7] J. Petremand, J. Puyal, J.Y. Chatton, J. Duprez, F. Allagnat, M. Frias, R.W. James, G. Waeber,

J.C. Jonas, C. Widmann, HDLs protect pancreatic beta-cells against ER stress by restoring protein folding and trafficking, Diabetes, 61 (2012) 1100-1111.

[8] W.S. Davidson, R.A. Silva, S. Chantepie, W.R. Lagor, M.J. Chapman, A. Kontush, Proteomic analysis of defined HDL subpopulations reveals particle-specific protein clusters: relevance to antioxidative function, Arterioscler Thromb Vasc Biol, 29 (2009) 870-876.

[9] N. Xu, B. Dahlback, A novel human apolipoprotein (apoM), J Biol Chem, 274 (1999) 31286-31290.

[10] X. Wu, N. Niu, K. Brismar, X. Zhu, X. Wang, S. Efendic, T. Du, Y. Liu, H.F. Gu, Apolipoprotein M promoter polymorphisms alter promoter activity and confer the susceptibility to the development of type 1 diabetes, Clin Biochem, 42 (2009) 17-21.

[11] N. Niu, X. Zhu, Y. Liu, T. Du, X. Wang, D. Chen, B. Sun, H.F. Gu, Single nucleotide polymorphisms in the proximal promoter region of apolipoprotein M gene (apoM) confer the susceptibility to development of type 2 diabetes in Han Chinese, Diabetes Metab Res Rev, 23 (2007) 21-25.

[12] J.W. Zhou, S.K. Tsui, M.C. Ng, H. Geng, S.K. Li, W.Y. So, R.C. Ma, Y. Wang, Q. Tao, Z.Y. Chen, J.C. Chan, Y.Y. Ho, Apolipoprotein M gene (APOM) polymorphism modifies metabolic and disease traits in type 2 diabetes, PLoS One, 6 (2011) e17324. 
[13] C. Wolfrum, M.N. Poy, M. Stoffel, Apolipoprotein M is required for prebeta-HDL

formation and cholesterol efflux to HDL and protects against atherosclerosis, Nat Med, 11

(2005) 418-422.

[14] C. Christoffersen, M. Jauhiainen, M. Moser, B. Porse, C. Ehnholm, M. Boesl, B. Dahlback,

L.B. Nielsen, Effect of apolipoprotein $\mathrm{M}$ on high density lipoprotein metabolism and

atherosclerosis in low density lipoprotein receptor knock-out mice, J Biol Chem, 283 (2008)

1839-1847.

[15] C. Christoffersen, H. Obinata, S.B. Kumaraswamy, S. Galvani, J. Ahnstrom, M. Sevvana, C.

Egerer-Sieber, Y.A. Muller, T. Hla, L.B. Nielsen, B. Dahlback, Endothelium-protective

sphingosine-1-phosphate provided by HDL-associated apolipoprotein M, Proc Natl Acad Sci U

S A, 108 (2011) 9613-9618.

[16] S. Spiegel, S. Milstien, The outs and the ins of sphingosine-1-phosphate in immunity, Nat

Rev Immunol, 11 (2011) 403-415.

[17] M. Maceyka, K.B. Harikumar, S. Milstien, S. Spiegel, Sphingosine-1-phosphate signaling and its role in disease, Trends Cell Biol, 22 (2012) 50-60.

[18] M. Kurano, K. Tsukamoto, R. Ohkawa, M. Hara, J. Iino, Y. Kageyama, H. Ikeda, Y. Yatomi, Liver involvement in sphingosine 1-phosphate dynamism revealed by adenoviral hepatic overexpression of apolipoprotein M, Atherosclerosis, (2013). 
[19] J. Miyazaki, K. Araki, E. Yamato, H. Ikegami, T. Asano, Y. Shibasaki, Y. Oka, K.

Yamamura, Establishment of a pancreatic beta cell line that retains glucose-inducible insulin secretion: special reference to expression of glucose transporter isoforms, Endocrinology, 127 (1990) 126-132.

[20] Y. Yatomi, Plasma sphingosine 1-phosphate metabolism and analysis, Biochim Biophys Acta, 1780 (2008) 606-611.

[21] M. Kurano, O.N. Iso, M. Hara, E. Noiri, K. Koike, T. Kadowaki, K. Tsukamoto, Plant sterols increased IL-6 and TNF-alpha secretion from macrophages, but to a lesser extent than cholesterol, J Atheroscler Thromb, 18 (2011) 373-383.

[22] S.G. Laychock, S.M. Sessanna, M.H. Lin, L.D. Mastrandrea, Sphingosine 1-phosphate affects cytokine-induced apoptosis in rat pancreatic islet beta-cells, Endocrinology, 147 (2006) $4705-4712$.

[23] J. Cantrell Stanford, A.J. Morris, M. Sunkara, G.J. Popa, K.L. Larson, S. Ozcan, Sphingosine 1-phosphate (S1P) regulates glucose-stimulated insulin secretion in pancreatic beta cells, J Biol Chem, 287 (2012) 13457-13464.

[24] K. Ueki, T. Okada, J. Hu, C.W. Liew, A. Assmann, G.M. Dahlgren, J.L. Peters, J.G. Shackman, M. Zhang, I. Artner, L.S. Satin, R. Stein, M. Holzenberger, R.T. Kennedy, C.R. Kahn, R.N. Kulkarni, Total insulin and IGF-I resistance in pancreatic beta cells causes overt 
diabetes, Nat Genet, 38 (2006) 583-588.

[25] I.B. Leibiger, B. Leibiger, P.O. Berggren, Insulin signaling in the pancreatic beta-cell, Annu Rev Nutr, 28 (2008) 233-251.

[26] Y. Takuwa, Y. Okamoto, K. Yoshioka, N. Takuwa, Sphingosine-1-phosphate signaling in physiology and diseases, Biofactors, 38 (2012) 329-337.

[27] K. Fujimoto, K.S. Polonsky, Pdx1 and other factors that regulate pancreatic beta-cell survival, Diabetes Obes Metab, 11 Suppl 4 (2009) 30-37.

[28] M. Cnop, F. Foufelle, L.A. Velloso, Endoplasmic reticulum stress, obesity and diabetes, Trends Mol Med, 18 (2012) 59-68.

[29] S.G. Fonseca, J. Gromada, F. Urano, Endoplasmic reticulum stress and pancreatic beta-cell death, Trends Endocrinol Metab, 22 (2011) 266-274.

[30] H.B. Brewer, Jr., Clinical review: The evolving role of HDL in the treatment of high-risk patients with cardiovascular disease, J Clin Endocrinol Metab, 96 (2011) 1246-1257.

[31] E.A. Podrez, Anti-oxidant properties of high-density lipoprotein and atherosclerosis, Clin Exp Pharmacol Physiol, 37 (2010) 719-725.

[32] Y. Yatomi, Sphingosine 1-phosphate in vascular biology: possible therapeutic strategies to control vascular diseases, Curr Pharm Des, 12 (2006) 575-587.

[33] H. Shimizu, F. Okajima, T. Kimura, K. Ohtani, T. Tsuchiya, H. Takahashi, A. Kuwabara, H. 
Tomura, K. Sato, M. Mori, Sphingosine 1-phosphate stimulates insulin secretion in HIT-T 15

cells and mouse islets, Endocr J, 47 (2000) 261-269.

[34] C. Wolfrum, J.J. Howell, E. Ndungo, M. Stoffel, Foxa2 activity increases plasma high density lipoprotein levels by regulating apolipoprotein M, J Biol Chem, 283 (2008) 16940-16949.

[35] N. Xu, B. Ahren, J. Jiang, P. Nilsson-Ehle, Down-regulation of apolipoprotein M expression is mediated by phosphatidylinositol 3-kinase in HepG2 cells, Biochim Biophys Acta, 1761 (2006) 256-260.

[36] T. Kimura, H. Tomura, C. Mogi, A. Kuwabara, A. Damirin, T. Ishizuka, A. Sekiguchi, M. Ishiwara, D.S. Im, K. Sato, M. Murakami, F. Okajima, Role of scavenger receptor class B type I and sphingosine 1-phosphate receptors in high density lipoprotein-induced inhibition of adhesion molecule expression in endothelial cells, J Biol Chem, 281 (2006) 37457-37467. [37] B.A. Wilkerson, G.D. Grass, S.B. Wing, W.S. Argraves, K.M. Argraves, Sphingosine 1-phosphate (S1P) carrier-dependent regulation of endothelial barrier: high density lipoprotein (HDL)-S1P prolongs endothelial barrier enhancement as compared with albumin-S1P via effects on levels, trafficking, and signaling of S1P1, J Biol Chem, 287 (2012) 44645-44653.

[38] E. Rapizzi, M.L. Taddei, T. Fiaschi, C. Donati, P. Bruni, P. Chiarugi, Sphingosine 1-phosphate increases glucose uptake through trans-activation of insulin receptor, Cell Mol Life 


\section{Figure Legends}

Figure 1. ApoM overexpression in wild-type mice prevented the increase in blood glucose levels after ad libitum feeding.

Ten-week-old male C57BL6 mice were treated with adenovirus coding apoM (apoM) or control adenovirus (null). After 5 days, an analysis was performed $(\mathrm{n}=5)$. (A) Plasma apoM levels. (B) Plasma S1P levels. (C) Fasting and ad libitum feeding blood glucose levels. (D) Insulin tolerance test; insulin ( $0.5 \mathrm{U} / \mathrm{kg}$ body weight) was administered intraperitoneally $(\mathrm{n}=5)$. (E) and (F) Western blot analysis of phosphorylated Akt (P-Akt) and total Akt (T-Akt) with homogenates of livers and skeletal muscles $(n=5)$.

Figure 2. ApoM overexpression enhanced insulin secretion, and this increment was inhibited by treatment with VPC 23019.

Ten-week-old male C57BL6 mice were treated with adenovirus coding apoM (apoM) or control adenovirus (null). From the second day, VPC 23019 (VPC; $0.75 \mathrm{mg} / \mathrm{kg}$ body weight) or vehicle was administered intraperitoneally. After 5 days, an analysis was performed $(n=5-6 /$ each group). (A) Fasting and ad libitum feeding blood glucose levels. null+V and apoM+V represents mice infected with control virus and apoM-overexpressing mice treated with VPC, respectively. ${ }^{*} P<0.05$ vs. null $+\mathrm{V},{ }^{* *} P<0.01$ vs. the other groups. (B-E) Glucose tolerance test; glucose (2 g/kg body weight) was administered intraperitoneally. (B) Time course of blood glucose. ${ }^{*} P<$ 
0.05 vs. null $+\mathrm{V}, * * P<0.01$ vs. the other groups, $\uparrow P<0.05$ vs. null, $P<0.01$ vs. null $+\mathrm{V}$, $\ddagger$

$P<0.01$ vs. null and null+V, $\S \mathrm{P}<0.05$ vs. null, \# $\mathrm{P}<0.05$ vs. null and null+V. (C) AUC of blood glucose. ${ }^{*} P<0.01$ vs. null and null $+\mathrm{V}$, ${ }^{* *} P<0.01$ vs. null and null $+\mathrm{V}, \mathrm{P}<0.05$ vs. apoM+V. (D) Time course of plasma insulin levels. $* P<0.01$ vs. the other groups. (E) AUC of plasma insulin levels, $* P<0.01$ vs. the other groups.

\section{Figure 3. ApoM-Lps increased insulin secretion from MIN6 cells.}

(A) Contents of albumin, apoA-I, and apoM in medium containing Null-Lps (left) or ApoM-Lps (right). (B, C) Time course of apoM and S1P levels in the media $(\mathrm{n}=3) . * P<0.01$ vs. null, $* * P$ $<0.01$ vs. the other group. (D, E) MIN6 cells were incubated in serum-free DMEM for 16 hours, and the media were replaced with media containing null-Lps (null) or apoM-Lps (apoM) with or without $5 \mu \mathrm{M}$ VPC $(\mathrm{V})$ and with or without $500 \mathrm{nM} \mathrm{S1P.} \mathrm{Then,} \mathrm{the} \mathrm{cells} \mathrm{were} \mathrm{further} \mathrm{incubated}$ for 4 hours (D) or 16 hours (E). Then, the media of the MIN6 cells were pre-incubated in glucose-free HEPES-Krebs buffer containing Lps and S1P corresponding to the concentration after 4 hours or 16 hours of the incubation of MIN6 cells for 30 minutes. Subsequently, the medium was changed for HEPES-Krebs buffer containing $20 \mathrm{mM}$ glucose and the insulin contents in the media were measured $(\mathrm{n}=6) . * P<0.01$ vs. null, null $+\mathrm{V}$, apoM, and S1P 500+V, $* * P<0.01$ vs. other groups. 
Figure 4. ApoM-Lps promoted the phosphorylation of Akt.

(A, B) MIN6 cells were incubated in serum-free DMEM for 16 hours and the media were replaced with media containing null-Lps (null), null-Lps with $500 \mathrm{nM} \mathrm{S1P}(\mathrm{S} 1 \mathrm{P})$, or media containing apoM-Lps (apoM) and further incubated for 1 hour, 4 hours, or 16 hours, then analyzed ( $\mathrm{n}=3$ ). (A) Western blots of phosphorylated Akt (P-Akt) and total Akt (T-Akt). (B) Ratio of P-Akt to T-Akt. The ratio was normalized to the ratio in CM-null at 1 hour. $* P<0.05$ vs. the other groups, $* * P<0.01$ vs. the other groups, $\uparrow P<0.05$ vs. null. (C-F) MIN6 cells were incubated in serum-free DMEM for 16 hours, and the media were replaced with media containing null-Lps (null), with or without $500 \mathrm{nM} \mathrm{S1P} \mathrm{(S1P} \mathrm{500),} \mathrm{apoM-Lps} \mathrm{(apoM),} \mathrm{with} \mathrm{or}$ without $5 \mu \mathrm{M}$ VPC (V) and further incubated for 4 hours $(C, D)$ or 16 hours $(E, F)(n=3)$. (C, E) Western blots of P-Akt and T-Akt. (D, F) Ratio of P-Akt to T-Akt. The ratio was normalized to the ratio in null. $* P<0.01$ vs. null and null $+\mathrm{V}, P<0.05$ vs. apoM+V, $* * P<0.01$ vs. the other groups, $\uparrow P<0.05$ vs. null.

\section{Figure 5. ApoM-Lps promoted the phosphorylation of AmpK.}

MIN6 cells were incubated in serum-free DMEM for 16 hours and the media were replaced with media containing null-Lps (null), with or without $500 \mathrm{nM} \mathrm{S1P} \mathrm{(S1P} \mathrm{500),} \mathrm{apoM-Lps}$ 
(apoM), with or without $5 \mu \mathrm{M}$ VPC (V) and further incubated for 4 hours (A, B) or 16 hours (C,

D) $(n=3)$. (A, C) Western blots of phosphorylated AmpK (P-AmpK) and total AmpK

(T-AmpK). (B, D) Ratio of P-AmpK to T-AmpK. The ratio was normalized to the ratio in null. $* P<0.05$ vs. null $+\mathrm{V}$, $* * P<0.05$ vs. null and apoM+V and $P<0.01$ vs. null $+\mathrm{V}, \dagger P<0.01$ vs. the other groups.

\section{Figure 6. ApoM-Lps increased the expression of Pdx-1.}

MIN6 cells were incubated in serum-free DMEM for 16 hours and the media were replaced with media containing null-Lps (null), with or without $500 \mathrm{nM} \mathrm{S1P}$ (S1P 500), apoM-Lps (apoM), with or without $5 \mu \mathrm{M}$ VPC (V) and further incubated for 4 hours (A, B, E) or 16 hours $(\mathrm{C}, \mathrm{D}, \mathrm{F})(\mathrm{A}-\mathrm{D} ; \mathrm{n}=3, \mathrm{E}$ and F; $\mathrm{n}=4)$. (A, C) Western blots of Pdx-1 and Actin. (B, D) Ratio of Pdx-1 to Actin. The ratio was normalized to the ratio in null. ${ }^{*} P<0.05$ vs. null and null+V, $P<$ 0.05 vs. apoM+V, $* * P<0.01$ vs. the other groups, $\uparrow P<0.05$ vs. null and null $+\mathrm{V}$. (E, F) $\mathrm{Pdx}-1$ mRNA level. GAPDH was utilized as a control. $* P<0.05$ vs. null $+\mathrm{V}, * * P<0.01$ vs. null+V and $P<0.05$ vs. apoM+V. 


\section{Figure 7. ApoM-Lps attenuated ER stress.}

MIN6 cells were incubated in serum-free DMEM for 16 hours and the media were replaced with media containing null-Lps (null), with or without $500 \mathrm{nM} \mathrm{S1P}$ (S1P 500), apoM-Lps (apoM), with or without $5 \mu \mathrm{M}$ VPC (V) and further incubated for 4 hours $(\mathrm{n}=4)$. (A, B) Ratio of non-spliced XBP1 to spliced XBP1. ${ }^{*} P<0.05$ vs. null+V, $* * P<0.01$ vs. null, null+V, apoM+V, and S1P 500+V, and $P<0.05$ vs. S1P 500, $\uparrow P<0.01$ vs. null, null+V, and S1P 500+V. (C) Grp78 mRNA level. GAPDH was utilized as a control. $* P<0.05$ vs. apoM+V.

Figure 8. Utilization of recombinant apoM as vehicle augmented S1P-mediated insulin secretion from MIN6 cells.

MIN6 cells were incubated in serum-free DMEM for 16 hours and the media were replaced with DMEM containing $0 \mathrm{nM}, 50 \mathrm{nM}$, and $500 \mathrm{nM}$ S1P, utilizing albumin or recombinant apoM as vehicle and further incubated for 4 hours. (A) S1P concentration in the medium after 4 hours of incubation $(\mathrm{n}=3)$. $* P<0.01$ vs. the other groups. (B) Insulin secretion from MIN6 cells investigated as Figure $3 \mathrm{C}(\mathrm{n}=6) .{ }^{*} P<0.05$ vs. $0 \mathrm{nM}$ albumin-bound $\mathrm{S} 1 \mathrm{P}, * * P<0.01$ vs. $0 \mathrm{nM}$ albumin-bound S1P and $500 \mathrm{nM}$ albumin-bound S1P with VPC, $\dagger P<0.01$ vs. $0 \mathrm{nM}$ and $50 \mathrm{nM}$ albumin-bound S1P and $0 \mathrm{nM}$ recombinant apoM-bound S1P and $50 \mathrm{nM}$ recombinant-bound S1P with VPC, $₫ P<0.01$ vs. $0 \mathrm{nM}, 50 \mathrm{nM}$ and $500 \mathrm{nM}$ albumin-bound $\mathrm{S} 1 \mathrm{P}$ and $0 \mathrm{nM}$ and 50 $\mathrm{nM}$ recombinant apoM-bound S1P and $500 \mathrm{nM}$ recombinant apoM-bound S1P with VPC. 


\section{Figure 1}
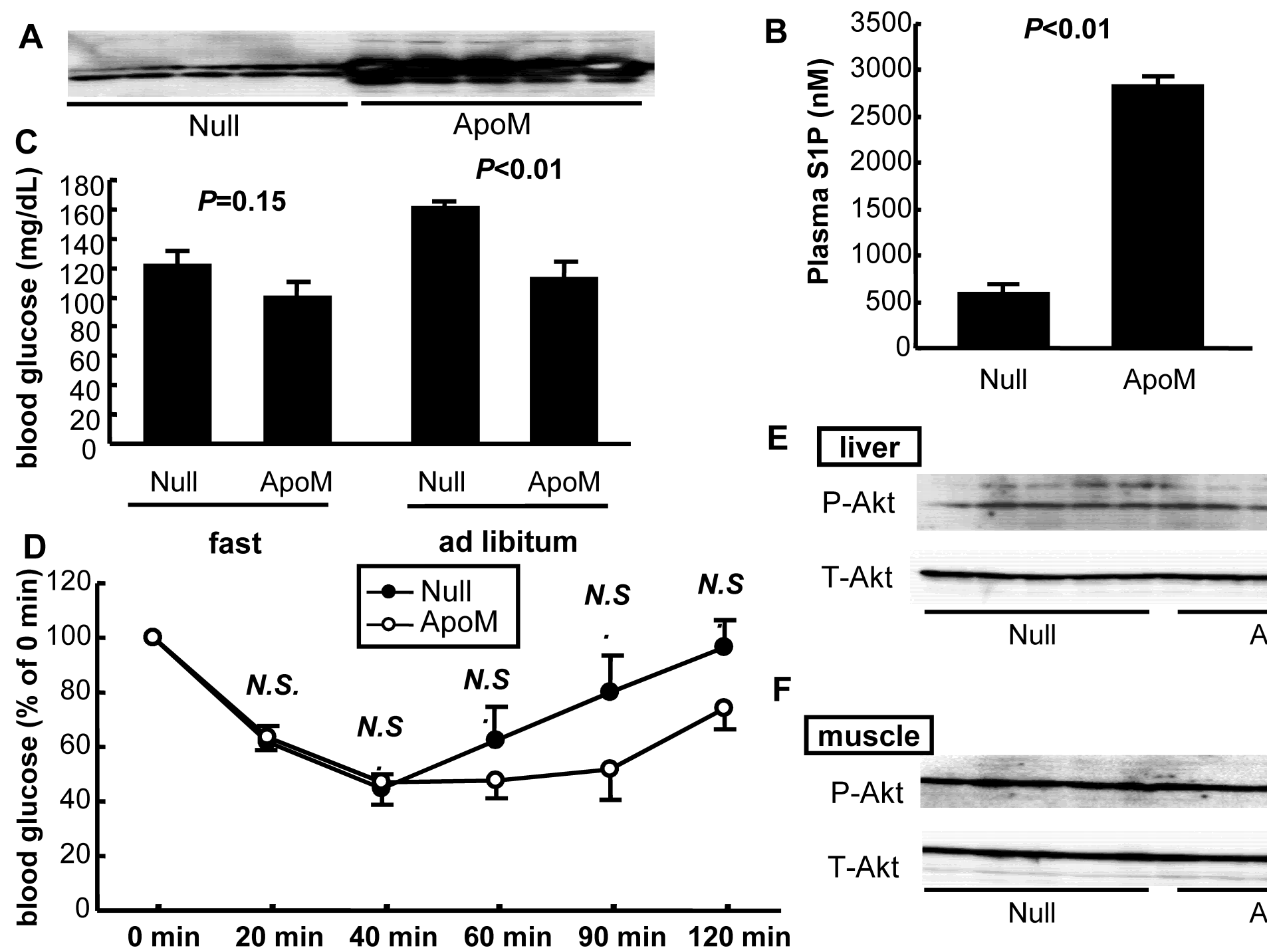

E liver

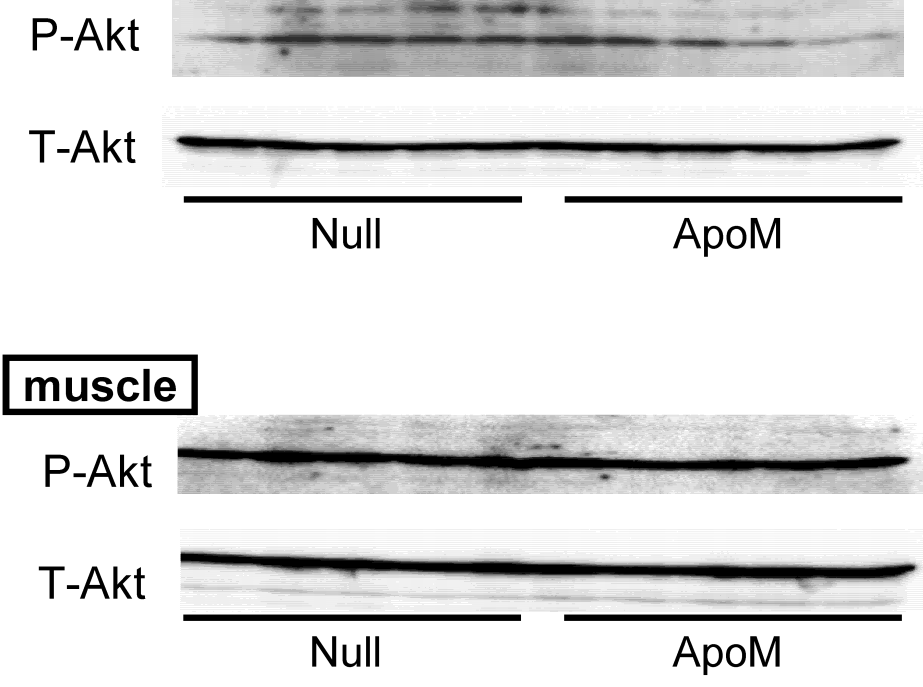


Figure 2
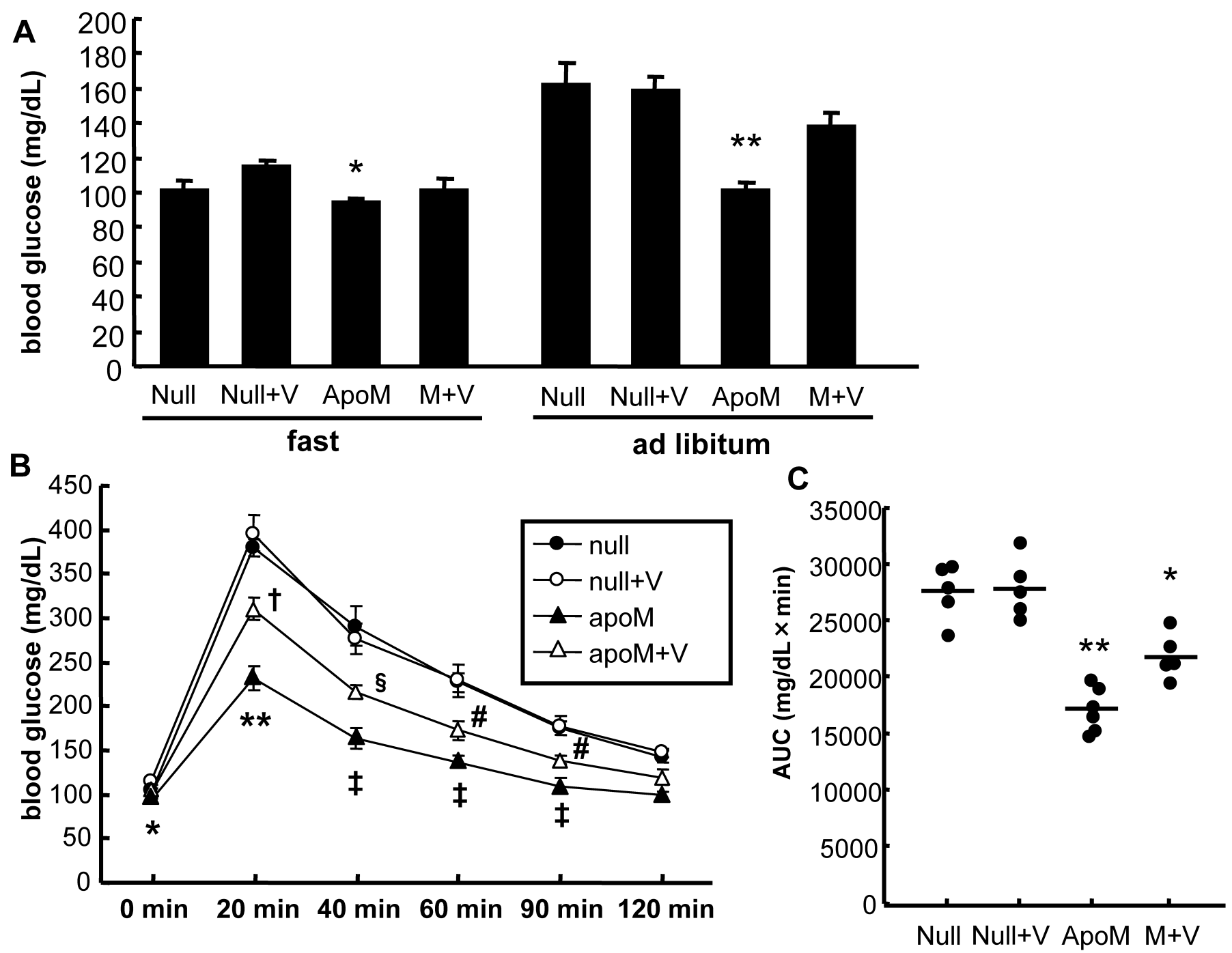

D

E
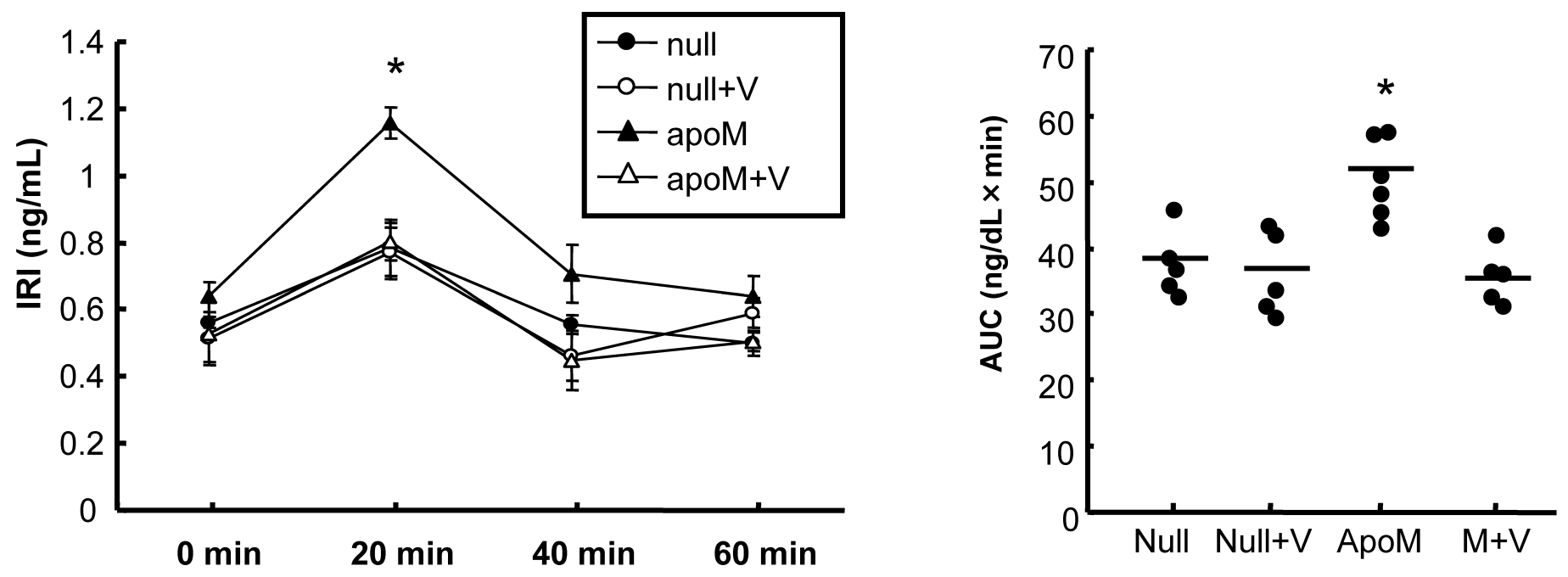
Figure 3

$\begin{array}{llllllllllllll}\text { A dilution } & \mathrm{x} 2 & \mathrm{X} 4 & \mathrm{x} 8 & \mathrm{X} 16 & \mathrm{X} 32 & \mathrm{X} 64 & \mathrm{X} 2 & \mathrm{X} 4 & \mathrm{x} 8 & \mathrm{X} 16 & \mathrm{X} 32 & \mathrm{X} 64\end{array}$

albumin

apoA1

apoM

$\begin{array}{lllllllllllll}\text { dilution } & \mathrm{X} 2 & \mathrm{X} 4 & \mathrm{X} 8 & \mathrm{X} 16 & \mathrm{X} 32 & \mathrm{X} 64 & \mathrm{X} 2 & \mathrm{X} 4 & \mathrm{X} 8 & \mathrm{X} 16 & \mathrm{X} 32 & \mathrm{X} 64\end{array}$

Null-Lps

B

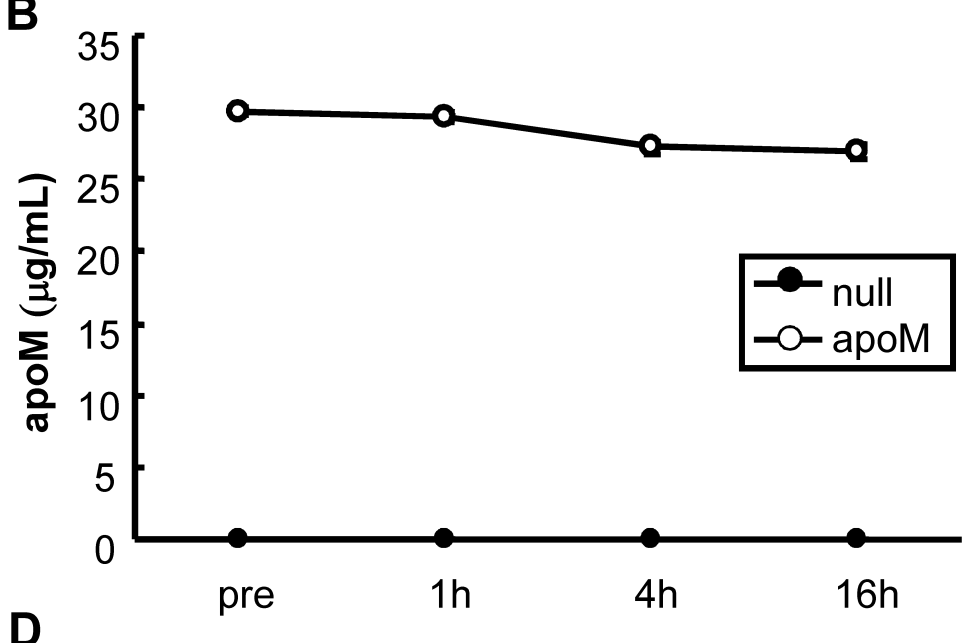

C
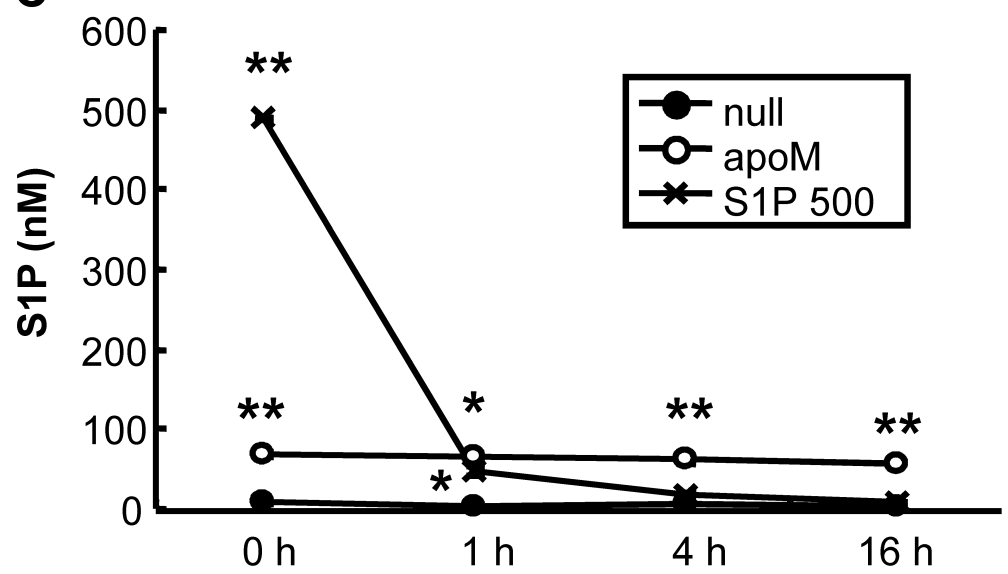

E

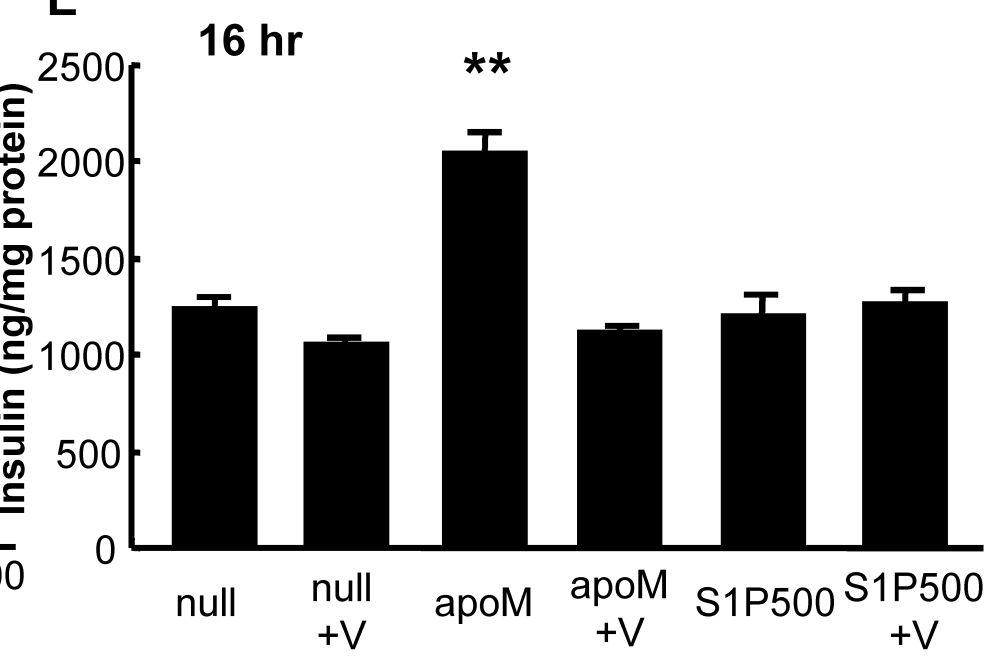


Figure 4

$A$

P-Akt

T-Akt

$\frac{1 \mathrm{~h} 4 \mathrm{~h} 16 \mathrm{~h}}{\text { null }} \frac{1 \mathrm{~h} \quad 4 \mathrm{~h} \quad 16 \mathrm{~h}}{\text { apoM }} \frac{1 \mathrm{~h} \quad 4 \mathrm{~h} \quad 16 \mathrm{~h}}{\operatorname{S1P} 500}$
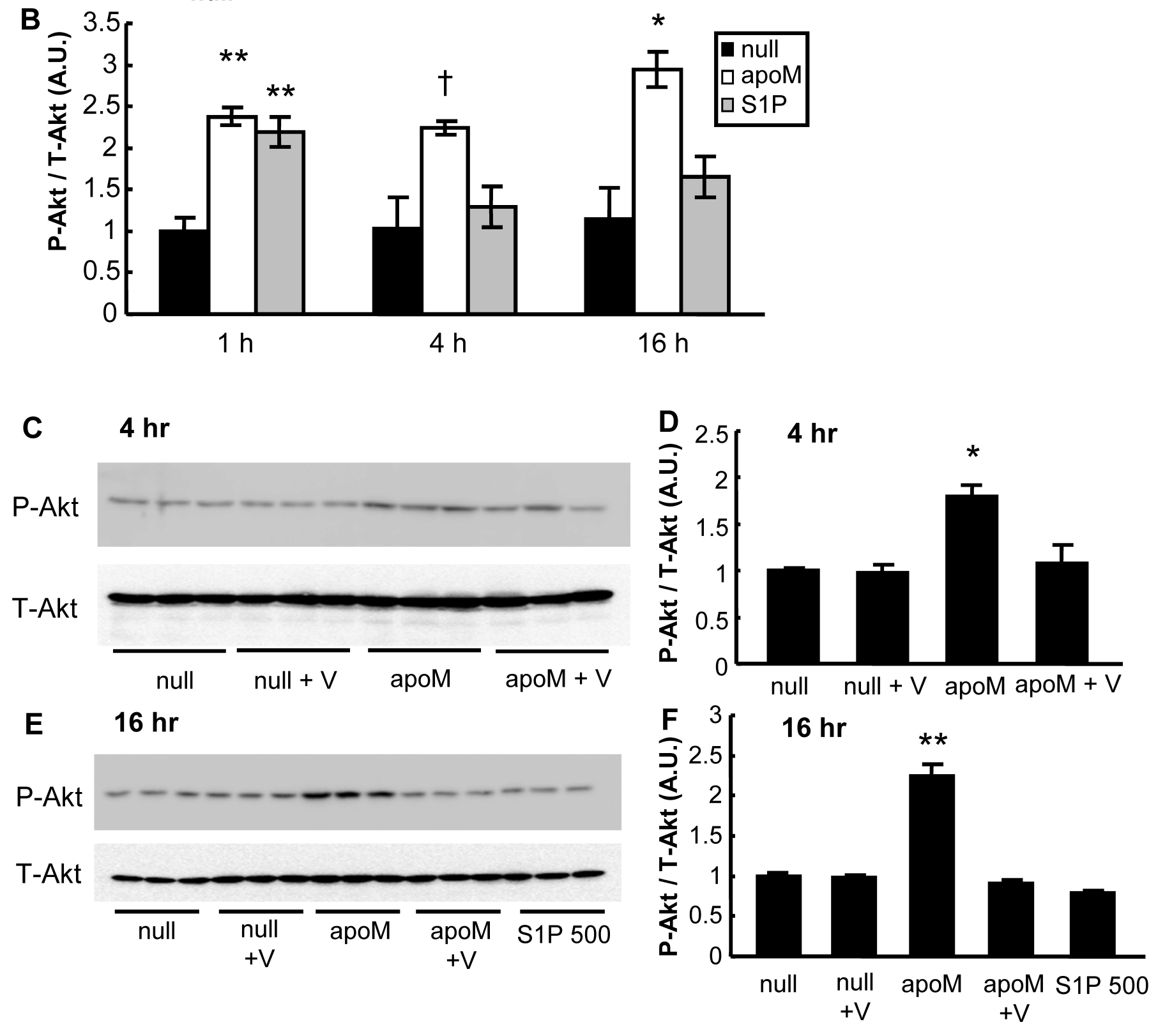
Figure 5

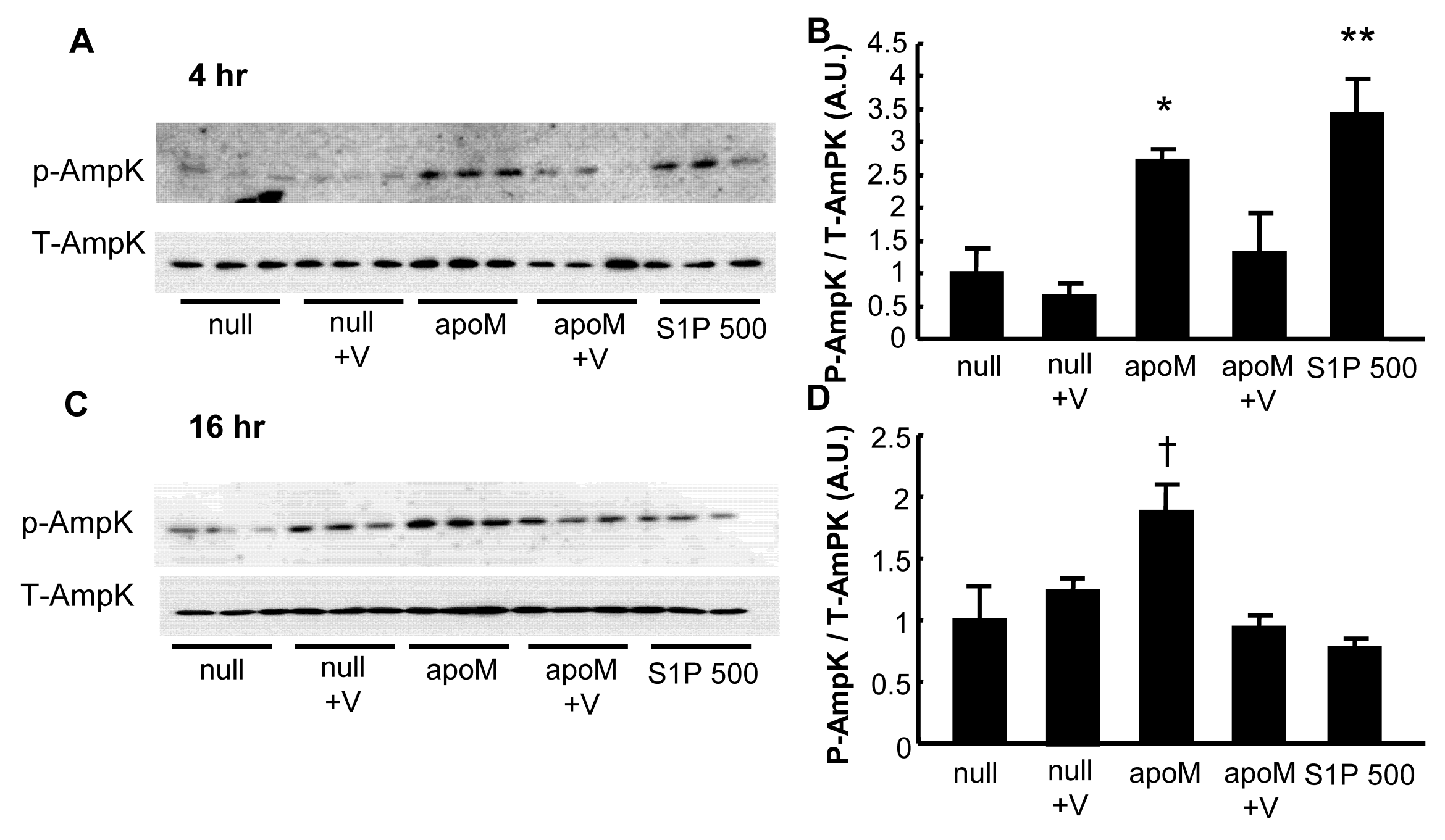


Figure 6
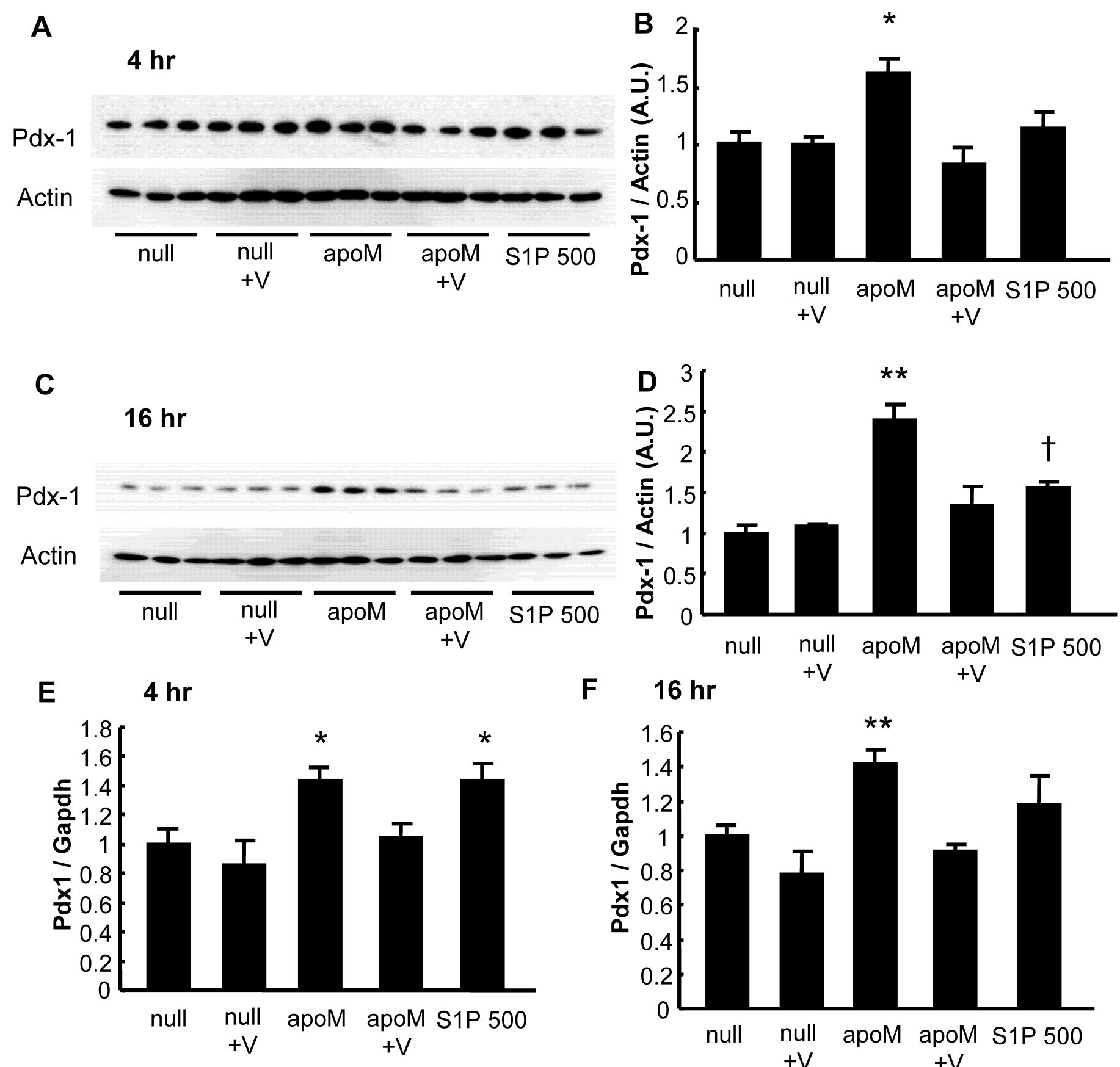

F

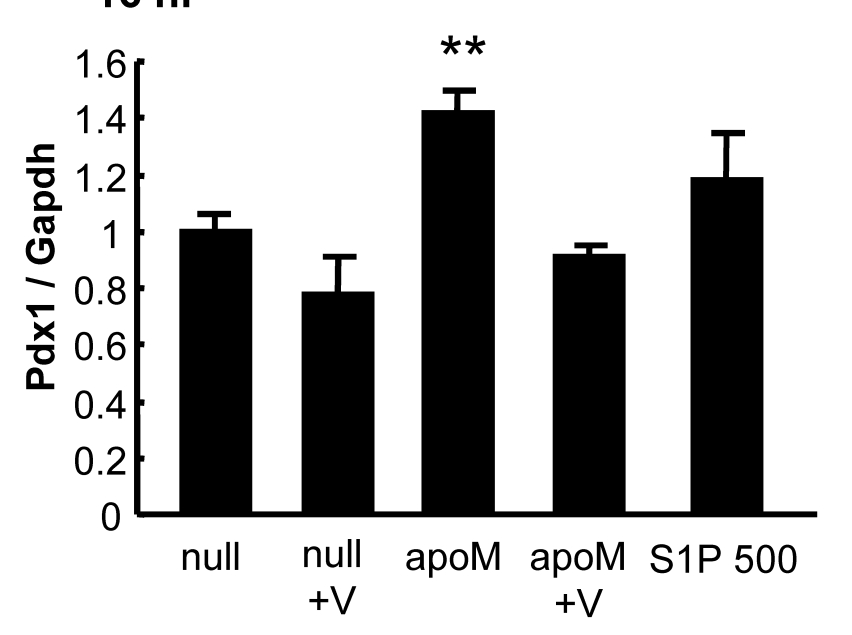


Figure 7
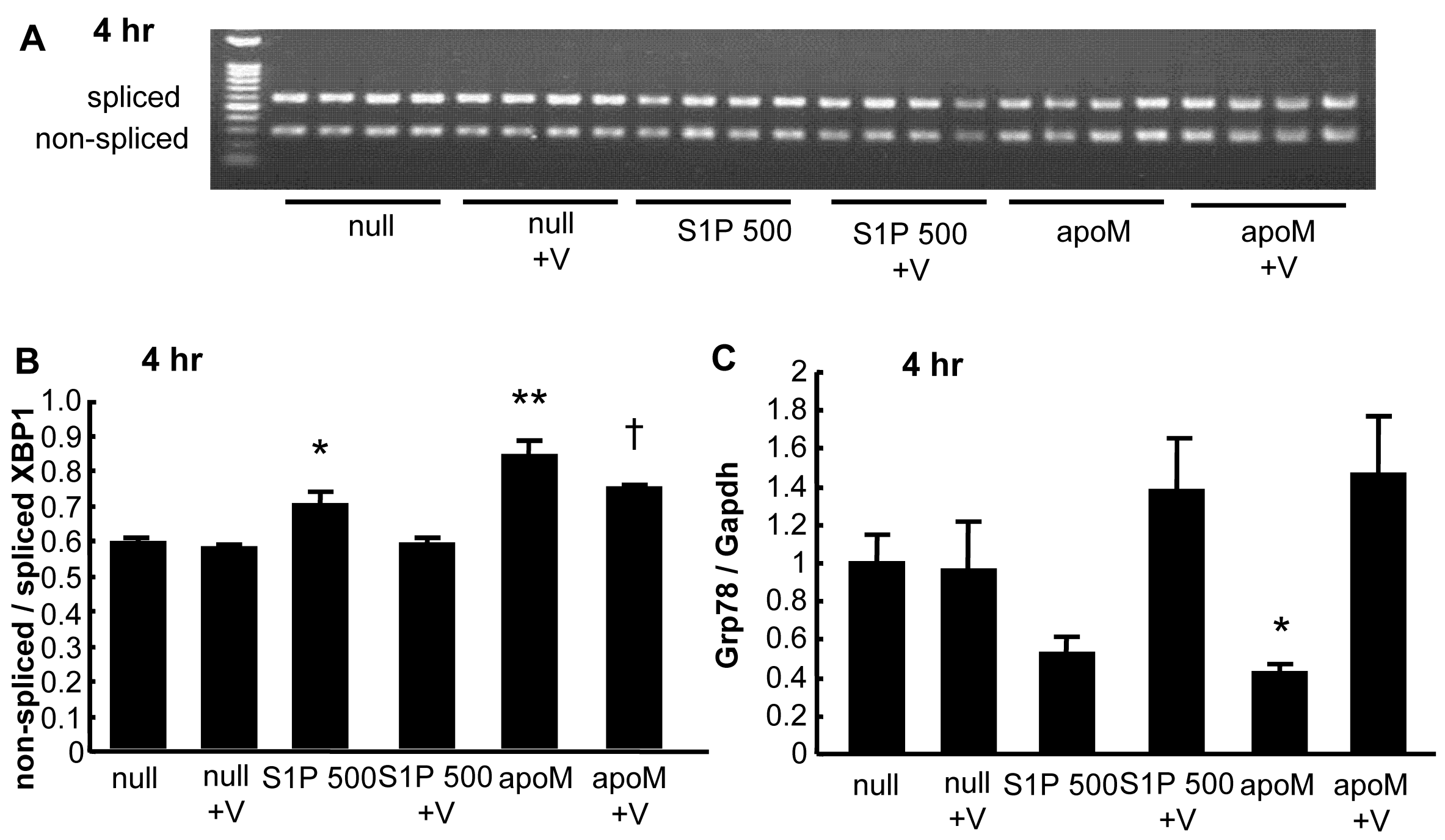
Figure 8

A

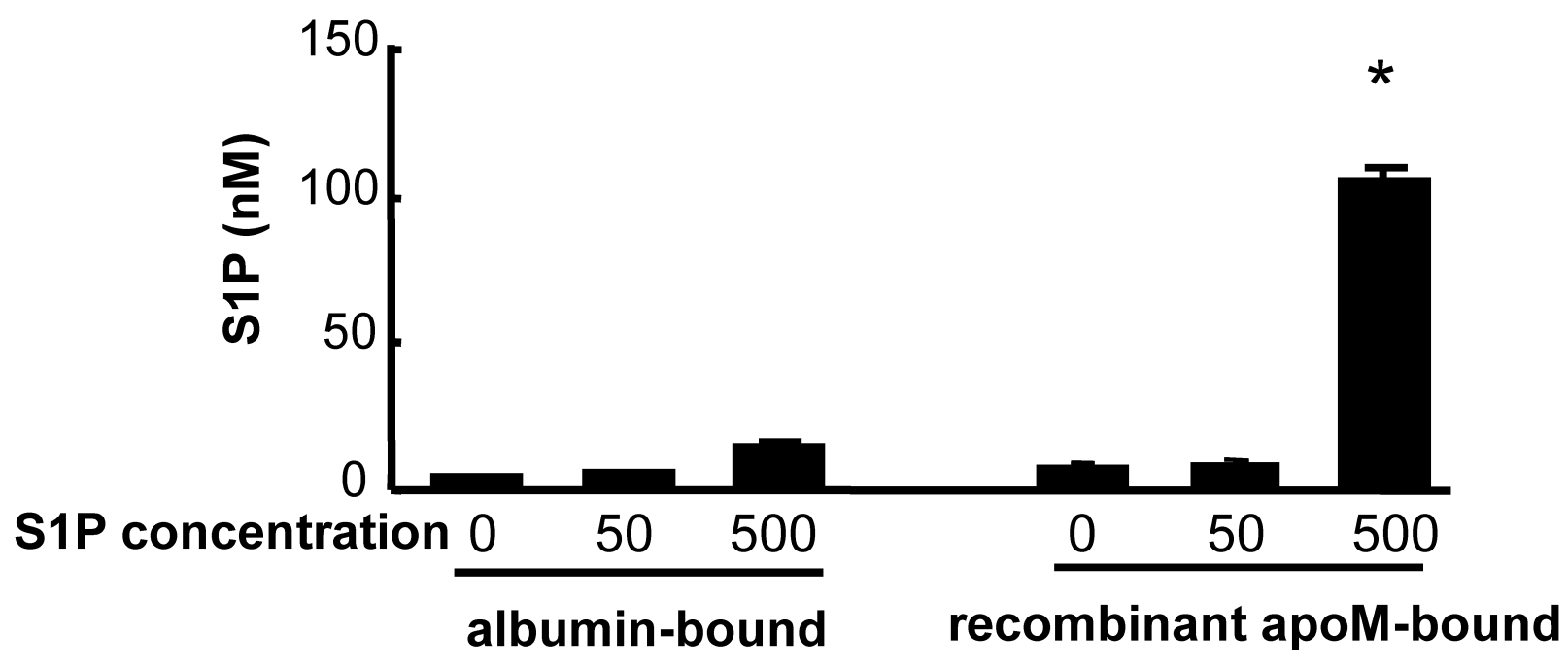

B

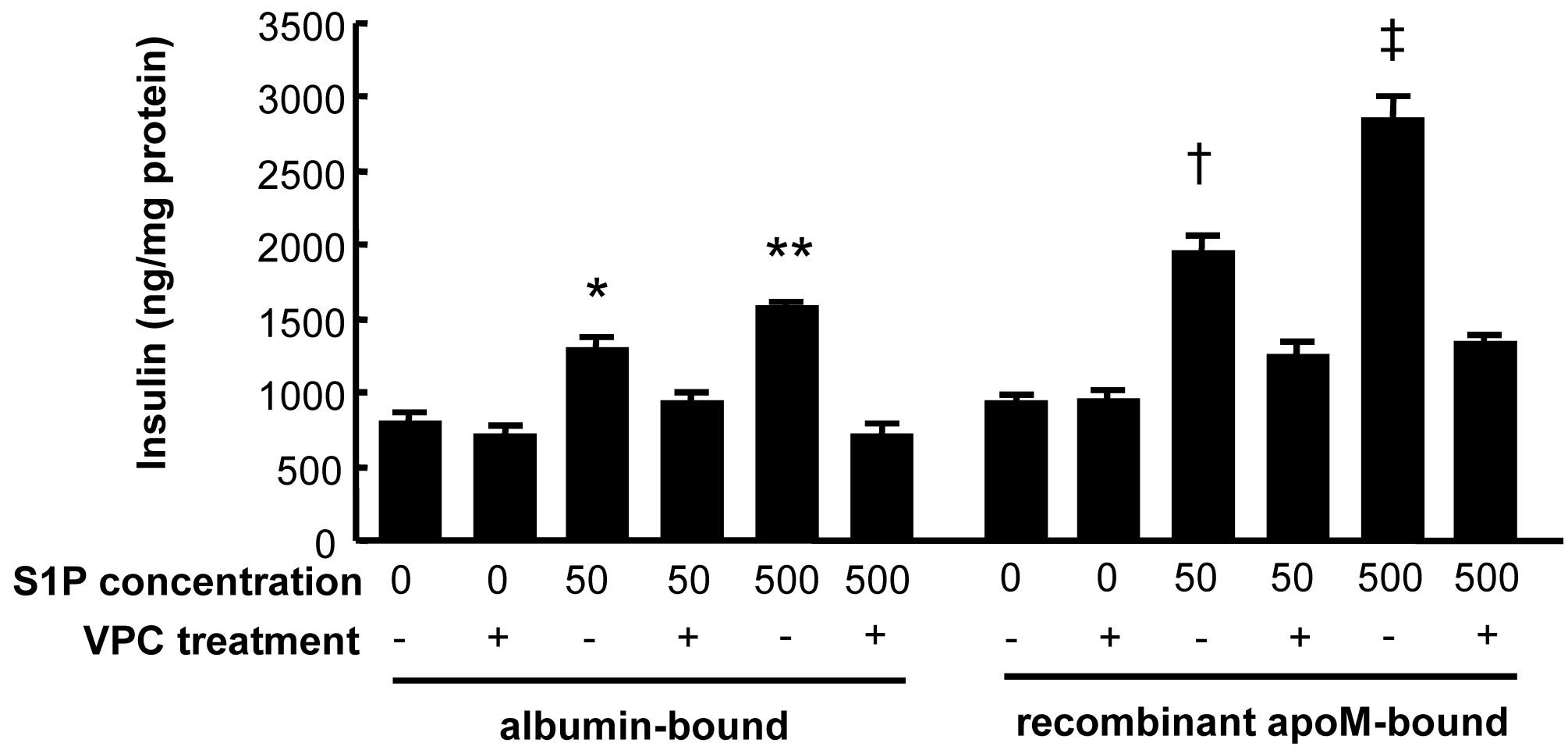

